\title{
Prevalence of Methicillin-Resistant Staphylococcus aureus Isolated from Clinical Samples at Narayani Samudayik Hospital, Chitwan, Nepal
}

\author{
Rama Adhikari ${ }^{1}$, Bindu Kshetri ${ }^{1}$, Khem Narayan Sharma ${ }^{2}$, Santosh Khanal ${ }^{1}$, Om Prakash Panta ${ }^{1}$, \\ Suprina Sharma ${ }^{1}$ Pramod Poudel $^{3}$ \\ ${ }^{1}$ Department of Microbiology, National College (Affiliated to Tribhuvan University), Khusibu, Nayabazar, \\ Kathmandu, Nepal \\ ${ }^{2}$ NPI-Narayani Samudayik Hospital, Chitwan \\ Central Department of Biotechnology, Tribhuvan University, Nepal
}

Corresponding author: Pramod Poudel, Central Department of Biotechnology, Tribhuvan University, Kirtipur, Nepal, E-mail: poudel.pm@gmail.com

\begin{abstract}
Objectives: The main objective of this study was to determine the prevalence of Methicillin Resistant Staphylococcus aureus (MRSA) and MDR bacteria isolated from various clinical specimens from the patients attending Narayani Samudayik Hospital, Chitwan

Methods: A cross-sectional study was carried in NPI-Narayani Samudayik Hospital, Chitwan from June to December 2017. Altogether, 3610 clinical specimens mainly pus, blood and urine were collected, streaked on Mannitol Salt Agar and Blood Agar and incubated at $37^{\circ} \mathrm{C}$ for 24 hours. The confirmed colonies of $S$. aureus were sub-cultured on Nutrient Agar. The antibiotic susceptibility pattern of all the isolates $S$. aureus were determined by Kirby Bauer disc diffusion method. Isolates resistant to cefoxitin $(30 \mathrm{mcg})$ were considered as MRSA.

Result: Among 3610 total clinical samples, $17.6 \%$ (635/3610) showed growth and 95(14.96\%) S. aureus were isolated. Higher number of S. aureus was isolated from pus sample (93.15\%). Out of 95 S. aureus isolates, $55(57.89 \%)$ were identified as MRSA while 40 (42.10\%) were MSSA. Vancomycin, ceftriaxone and chloramphenicol were found to be most effective antibiotic against isolates. Whereas, the least effective antibiotic was cefoxitin followed by amoxiclav, oxacillin and amoxicillin.

Conclusion: This study concludes that the overall prevalence of MRSA and MDR among the bacterial isolates is higher compared to other studies. So, it is recommended to monitor the antibiotic susceptibility pattern of pathogens regularly and study the epidemiology of such isolates.
\end{abstract}

Keywords: MRSA, Staphylococcus aureus, vancomycin, cefoxitin

\section{INTRODUCTION}

Staphylococcus aureus has emerged as one of the most important human pathogens, and has over the past several decades, been a leading cause of hospital and community - acquired infections (Lowy 1998). Methicillin, originally called celbenine, is a semisynthetic derivative of penicillin which is chemically modified to tolerate the degradative action of penicillinase. S.aureus that are resistant to methicillin

Date of Submission: September 16, 2020

Published Online: December, 2020 or oxacillin, the penicillinase stable $\beta$-lactam antibiotic, are known as Methicillin resistant $S$. aureus i.e MRSA. MRSA grouped under HA- MRSA (Healthcare associated methicillin resistant S.aureus) and CAMRSA (Community acquired methicillin resistant S.aureus) (Giacometti et al. 2000).

Methicillin resistance arises following the inactivation of beta lactamase enzymes, acquisition of nobel DNA, which results in production of a new penicillin-binding

Date of Acceptance: November 6, 2020

DOI: https:/ / doi.org/10.3126/tujm.v7i0.33794 
protein (PBP), known as $\mathrm{PBP2}$ or $\mathrm{PBP} 2 \mathrm{a}$, which has low binding affinity for methicillin and other currently available $\beta$-lactams (Deleo et al. 2010).

The frequency of infection caused by MRSA has been significantly increased in last 10 years (Stapheton and Taylor 2002) Studies conducted in Nepal showed that the prevalence of MRSA was astronomically increased from 29.1\% to 61.6\% in between 1990 and 2003 (Moran et al. 2006 \& Khanal and Jha 2010). Lack of effective antibiotic policy for the proper use of antibiotic has resulted to the emergence of resistant strains and use of incomplete course of antibiotic without proper prescription is one of the leading causes of dissemination of antibiotic resistant (Khanal et al. 2003). Most studies on $S$. aureus have been conducted on sample from nose and throat but only a limited number of studies have been reported on $S$. aureus from pus sample.

This study was carried out to know the recent status of Methicillin Resistant S. aureus in Chitwan district, Nepal as many studies done in Nepal suggest the gradual emergence of MRSA in hospital (Mishra 2013). According to Karki et al. (2019) and Shrestha et al. (2018), prevalence of MRSA was reported as $26.4 \%$ and $16.7 \%$ respectively in different Nepalese hospital settings. The information obtained from this study helps to guide the clinicians in choosing appropriate antibiotics and prevent the emergence of resistance to the drug which are still sensitive. Findings can be used to determine trends in antibiotic susceptibilities and guide in formulation of local antibiotic policy.

\section{MATERIALS AND METHODS}

Clinical sample from June to December 2017 received in clinical microbiology lab of Narayani Samudayik Hospital, Chitwan were processed and all S. aureus isolates were included in the study. Ninety-five isolate of $S$. aureus were collected from culture sample received from different department of the hospital. The isolates were consecutive and non-repetitive (one per patient). One sample from one patient was inclusion criteria of study data. Second sample from other site of same patient was not considered for study.

\section{Inclusion and exclusion criteria}

Only those samples which were adequately collected and properly labeled were included in the study. Those samples which were not collected by medical officer or an experienced nurse or self-collected by patients were not included in the study.

\section{Sample collection, transportation and processing}

All the samples were collected by medical personnel using aseptic procedures. Pus samples were collected using sterile syringe. Blood samples were collected using sterile syringe ( $3 \mathrm{ml}$ from children and $5 \mathrm{ml}$ from adults) into a sterile blood collection bottle containing BHI. For urine, a wide-mouthed sterile leak-proof container was provided for collection. About 10-15 ml of midstream urine was collected (Cheesbrough 2006). All the samples were properly labelled and transferred to microbiology laboratory for further processing (Mahon et al. 2014).

Samples were culture on blood agar, Mannitol salt agar, MacConkey agar for 24 hours. Blood culture was inoculated in Brain Heart Infusion broth and sub culture on 24 and 72 hour on BA and MSA. Identification of organism was carried out by standard laboratory operating procedure (Gram Staining, Catalase test, Coagulate test).

The antibiotic susceptibility pattern of all the strains was determined by modified Kirby Bauer Disc diffusion method against the following antibiotic: amoxiclav (30 mcg), cefoxitin (30 mcg), chloramphenicol (30 $\mathrm{mcg})$, Tetracycline (30 mcg), vancomycin (30 mcg), erythromycin (15 mcg), gentamycin $(30 \mathrm{mcg})$ and ceftriaxone $(30 \mathrm{mcg})$. Screening for methicillin resistance was performed by cefoxitin disc diffusion method and interpreted according to CLSI guidelines (CLSI 2014). Briefly, isolates with zone of inhibition (ZOI $\geq 22 \mathrm{~mm}$ ) were identified as methicillin-susceptible (MSSA) and isolates with $\mathrm{ZOI} \leq 21 \mathrm{~mm}$ identified as methicillinresistant (MRSA). As a reference strain, S. aureus ATCC 25923 was used in this study. The obtained data were analyzed using the Microsoft Excel 2010.

\section{RESULTS}

Out of 3610 clinical samples, 635 (17.59\%) showed bacterial growth. Among them, 465 (73.2\%) were Escherichia coli and only $95(14.96 \%)$ isolates were identified as $S$. aureus. Other identified isolates were Pseudomonas spp., Klebsiella pneumoniae, Enterococcus spp. and coagulase negative Staphylococci. The majority of $S$. aureus were isolates from pus (59.13\%) followed by blood (10\%) (Table 1). 
Table 1: Distribution of S. aureus in clinical samples

\begin{tabular}{lcccc}
\hline Clinical samples & $\begin{array}{c}\text { Total no. of } \\
\text { samples }\end{array}$ & $\begin{array}{c}\text { Culture positive cases } \\
\text { N (\%) }\end{array}$ & $\begin{array}{c}\text { S. aureus isolated from } \\
\text { culture positive cases N (\%) }\end{array}$ & $\begin{array}{c}\text { S. aureus from total } \\
\text { samples N (\%) }\end{array}$ \\
\hline Pus & 200 & $115(62.8 \%)$ & $68(59.13 \%)$ & $34 \%$ \\
Blood & 1350 & $50(3.57 \%)$ & $5(10 \%)$ & $10 \%$ \\
Urine & 2060 & $512(24.85 \%)$ & $22(4.29 \%)$ & $1.06 \%$ \\
\hline Total & 3610 & $635(17.59 \%)$ & $95(14.96 \%)$ & $2.63 \%$ \\
\hline
\end{tabular}

$N$; Number of isolates

A total of 635 positive cases were obtained consisting comprising 41 females and 54 males were isolated. The 285 females and 350 males. Out of this, 95 S. aureus isolates in females were $14.38 \%$ and that in males were $15.42 \%$ (Table 2).

Table 2: Distribution of S. aureus in male and female patients

\begin{tabular}{|c|c|c|c|}
\hline Gender & S. aureus N (\%) & Other than S. aureus $\mathbf{N} \%)$ & Total N (\%) \\
\hline Female & $41(14.38 \%)$ & $244(85.61 \%)$ & $285(44.88 \%)$ \\
\hline Male & $54(15.42 \%)$ & $296(84.57 \%)$ & $350(55.12 \%)$ \\
\hline Total & 95 (14.96\%) & 540 (85.04\%) & 635 (100\%) \\
\hline
\end{tabular}

As described in figure 1, higher number of isolates were obtained from the age group <10, 30-39, 40-49 years with an incidence of $27.36 \%, 15.78 \%$ and $13.68 \%$ respectively. Few isolates were from age group above 70 years $(2.10 \%)$.

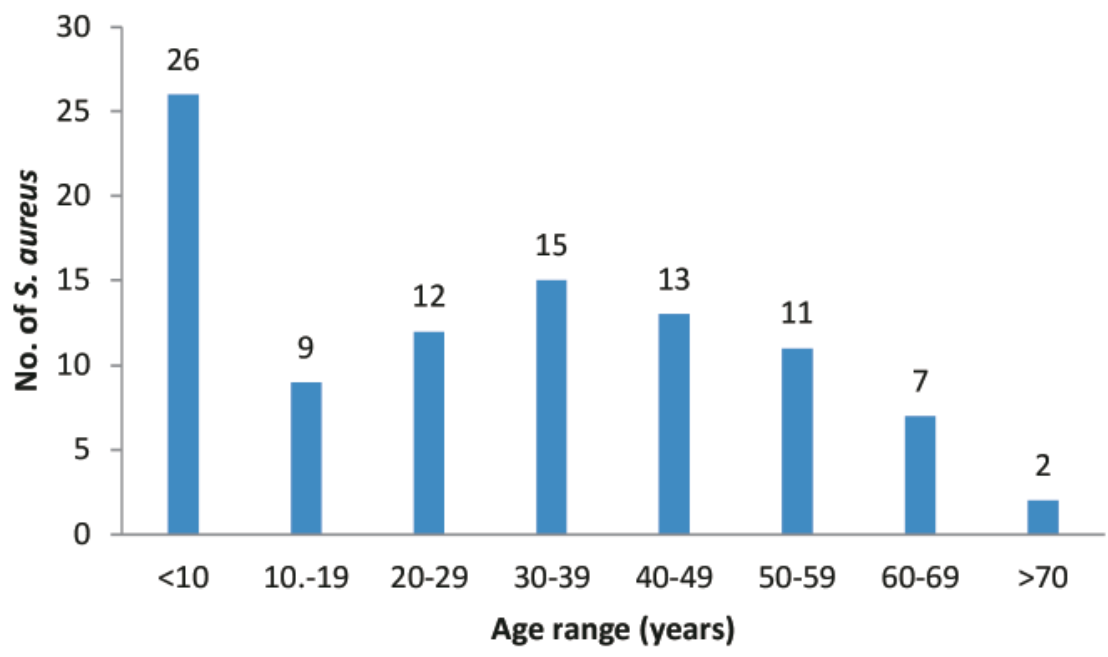

Figure 1: Age-wise distribution of patients with Staphylococcus aureus isolates

Fifty-five isolates were found to be MRSA. As shown in Table 3, out of 55 MRSA isolates 46 (83.64\%) were
MDR. Only 5 (12.5\%) among 40 (42.10\%) MSSA were MDR (Table1).

Table 3: MDR pattern among $S$. aureus isolates

\begin{tabular}{lccc}
\hline \multicolumn{1}{c}{ Drug resistance } & MRSA N (\%) & MSSA N (\%) & Total \\
\hline MDR & $46(83.64 \%)$ & $5(12.5 \%)$ & 51 \\
Non- MDR & $9(16.36 \%)$ & $35(87.5 \%)$ & 44 \\
\hline Total & $\mathbf{5 5 ( 1 0 0 \% )}$ & $\mathbf{4 0 ( 1 0 0 \% )}$ & $\mathbf{9 5}$ \\
\hline
\end{tabular}


The antimicrobial susceptibility pattern of $S$. aureus are summarized in Table 4. Out of 95 isolates of $S$. aureus, $55(57.89 \%)$ were MRSA. Most of the S. aureus isolates were sensitive to ceftriaxone $85(89.47 \%)$, vancomycin $83(87.36 \%)$ and chloramphenicol 71 (74.73\%). So, these were the more effective anti-staphylococcal drugs. Most of them were resistant to amoxiclav 70 (73.68\%) followed by amoxycillin 60 (63.15\%), cefoxitin (57.89\%) and oxacillin 52 (54.73\%).

Table 4: Antibiotic susceptibility patterns of $S$. aureus $(n=95)$

\begin{tabular}{lccc}
\hline \multirow{2}{*}{ Antibiotic } & \multicolumn{3}{c}{ Susceptibility pattern (n=95) } \\
\cline { 2 - 4 } & Sensitive N (\%) & Intermediate N (\%) & Resistant N (\%) \\
\hline Amoxiclav & $25(26.31 \%)$ & - & $70(73.68 \%)$ \\
Amoxycillin & $15(15.78 \%)$ & $20(21.05 \%)$ & $60(63.15 \%)$ \\
Cefoxitin & $40(42.10 \%)$ & - & $55(57.89 \%)$ \\
Ceftriaxone & $85(89.47 \%)$ & $3(3.15 \%)$ & $7(7.36 \%)$ \\
Chloramphenicol & $71(74.73 \%)$ & $20(21.05 \%)$ & $4(4.21 \%)$ \\
Ciprofloxacin & $65(67.36 \%)$ & $7(7.36 \%)$ & $23(24.21 \%)$ \\
Erythromycin & $47(49.47 \%)$ & - & $48(50.52 \%)$ \\
Gentamicin & $29(30.52 \%)$ & $22(23.15 \%)$ & $44(46.31 \%)$ \\
Levofloxacin & $32(33.68 \%)$ & $25(26.31 \%)$ & $38(40 \%)$ \\
Oxacillin & $36(37.89 \%)$ & $6(6.31 \%)$ & $52(54.73 \%)$ \\
Tetracycline & $69(72.63 \%)$ & - & $14(14.73 \%)$ \\
Vancomycin & $83(87.36 \%)$ & $10(10.52 \%)$ & $2(2.10 \%)$ \\
\hline
\end{tabular}

\section{DISCUSSION}

MRSA has emerged as a serious public health problem globally as it has the ability to acquire antimicrobial resistance over time, and it will continue to be a problem in the future. Today, most of the MRSA are multi-drug resistant i.e., resistant to a number of drugs, thus causing a clinical problem as antibiotic treatment becomes useless. Present study showed prevalence rate of MRSA to be $57.89 \%$. The study done in Kathmandu valley reported $44.9 \%$ as MRSA from nosocomial S. aureus (Shrestha et al. 2009). Rajbhandari et al. (2008) also reported 54.9\% MRSA isolates in Bir Hospital. MRSA was isolated at the rate 75.5\% from clinical samples in a study conducted by Rijal et al. in Pokhara Valley (Rijal et al. 2008). Similar study done in western parts of Nepal by Tiwari et al. (2009) also reported alarming high rate of MRSA isolate (69.1\%) which the authors has attributed to indiscriminate use of antibiotics and its accessibility in these.

Above studies show considerable variations between institutions, often in the same geographical areas, exist, demonstrating that MRSA prevalence, in some settings, significantly exceeds previous estimate. There could be many explanations for these differences: infection control measures, antibiotic prophylaxis and treatments used in each ward/hospital and, not less important, the clonal and often epidemic nature of these microorganisms (Betty et al. 2002).

Present study also shows maximum number of S. aureus and MRSA isolation from pus $(48 / 55)$ ascertaining the role of the organism as cause of pyogenic infection. Kumari et al. and Pandey et al. have also reported that the isolation of S. aureus is higher from pus samples (Kumari et al. 2008; Pandey et al. 2012).

Most of the studies suggest that tests with cefoxitin are more reliable those with oxacillin because cefoxitin is a potent inducer of the mecA regulatory system and widely used as a surrogate marker for detection of mecA genemediated methicillin resistance (Aliberti et al. 2016). In the present study the MRSA isolates showed a highest level of resistance towards cefoxitin (100\%), amoxiclave (83.63\%), erythromycin (52.72\%) and gentamycin (45.45\%). Tetracycline have excellent tissue penetration and demonstrate good staphylococcal activity at clinically achievable levels with a reported cure rate of $83 \%$ in MRSA skin and soft tissue infections (Idrees et al. 2009). The present study shows $89.47 \%$ of $S$. aureus being sensitive to ceftriaxone, $87.36 \%$ to vancomycin and $74.73 \%$ to tetracycline. This roughly correlates with the finding of Thapa et al. (2008) who have reported $73.84 \%$ of $S$. aureus as sensitive to tetracycline. 
The multi-drug resistant phenotype is a particular characteristic of the methicillin-resistant $S$. aureus strains. It has added to the burden of hospital personnel to control infection associated with MDR-MRSA. Present study shows alarmingly high rate of MDR strain among MRSA isolates (83.64\%). Similar studies have reported MDR-MRSA to be as 100\% (Kayastha 2010), 92\% (Thapa 2011), 75.86\% (Pandey et al. 2012). Indian literature also shows the isolation of MDRMRSA as high as 72.1\% (Tiwari et al. 2008).

Present study shows alarmingly high rate of MDR strain among MRSA isolates (90.19\%). The result is consistent with the previous reports in which MDR-MRSA isolates were confirmed as $100 \%$ (Kayastha 2010), 92\% (Thapa 2011) and 75.86\% (Pandey et al. 2012) and 93.1\% (Karki et al. 2019) from clinical samples. According to Gopalakrishnan (2010) the incidence of MRSA varied from $25 \%$ to $50 \%$ in India.

Though these MDR strains are not found with additional virulence properties, their characteristics multidrug resistance restricts the option available to treat infections caused by this organism (Voss and Doebbeling 1995).

\section{CONCLUSION}

The prevalence of S. aureus was found to be $2.63 \%$ and majority of them were sensitive to ceftriaxone. More than half of $S$. aureus were found to be MRSA and among them, $83.64 \%$ were found to be MDR. MRSA infection is still one of the most life-threatening infections as such infections are difficult to treat. Further detection and molecular characterization of the gene (mec A), phage typing and analyses of the plasmids of MRSA is necessary.

\section{ACKNOWLEDGEMENTS}

We are thankful to staffs of Narayani Samudayik Hospital, Chitwan and the patients for their cooperation during this study.

\section{CONFLICT OF INTEREST}

The authors declare that they have no conflict of interest.

\section{REFERENCES}

Aliberti S, Reyes LF, Faverio P, Sotgiu G, Dore S, Rodriguez AH, Soni NJ, Restrepo MI, Aruj PK, Attorri S and Barimboim E (2016) Global initiative for methicillin-resistant Staphylococcus aureus pneumonia (GLIMP): an international, observational cohort study. Lancet Infect Dis 16: 1364-1376.

Betty AF, Daniel FS, Alice SW (2002) Staphylococcus, Micrococcus and Similar Organisms. In: Baily and scott's Diagnostic Microbiology, $11^{\text {th }}$ edition (Mosby Inc: St.Louis).p284.

CLSI (2014) Performance standards for antimicrobials susceptibility testing. Clinical and Laboratory Standards Institute 950 West Valley Road, Suite 2500 Wayne, PA 19087 USA pp. 116- 122.Clinical and laboratory standards standards Institute.

Deleo FR, Otto M, Kreiswirth BN and chambers HF (2010) Community-associated methicillinresistant Staphylococcus aureus. Lancet 375:15571568.

Giacometti A, Cirion O and Schimizzi A (2000) Epidemiology and Microbiology of surgical wound infection. J Clinical Microbilogy 38: 918-922.

Gopalakrishnan R, Sureshkumar D. Changing trends in antimicrobial susceptibility and hospital acquired infections over an 8 year period in a tertiary care hospital in relation to introduction of an infection control programme (2010). J Assoc Physicians India 58: 25-31.

Idrees F, Jabeen K, Khan MS, Zafar A (2009) Antimicrobial resistance profile of Methicillin Resistant Staphylococcus aureus from skin and soft tissue isolates. J Pak Med Assoc 59: 266-269.

Karki S, Sah AK, Lamichhane J, Maharjan A, Sharma L, Rajbhandari R, Parajuli S, Acharya S and Khanal S (2019) Biofilm Formation and Detection of icaD Gene in Staphylococcus aureus Isolated from Clinical Specimens. Open Microbiol J, 13: 230-235.

Kayastha BB (2010) Methicillin resistant Staphylococcus aureus (MRSA) in different clinical samples from patients presenting at Kathmandu Model Hospital. M.Sc. Dissertation Submitted to Central Department of Microbiology, Tribhuwan University.

Khanal L and Jha B (2010) Prevalence of methicillin resistant Staphylococcus aureus (MRSA) among skin infection cases at a hospital in Chitwan, Nepal. Nepal Medical College Journal 12: 224-228.

Khanal LK, Adhikari RP and Guragain A (2018) Prevalence of Methicillin Resistant Staphylococcus 
aureus and Antibiotic Susceptibility Pattern in a Tertiary Hospital in Nepal. J Nepal Health Research Counc 16:172-174.

Kumari N, Mogapatra TM and Singh YI (2008) Prevalence of Methicillin- Resistant Staphylococcus aureus (MRSA) in a Tertiary-Care Hospital in Eastern Nepal. J Nepal Med Assoc 47: 53-56.

Lowy FD (1998) Staphylococcus aureus Infections. N Engl J Med 339: 520- 532.

Mahon CR, Lehman DC and George M (2014). Textbook of Diagnostic Microbiology 5ed: Elsevier

Mishra A (2013) Nasal carriage of Methicillin-resistant Staphylococcus aureus among patients visiting national kidney centre. M.S.c Dissertation submitted to Central Department of Microbiology, pp 4.

Moran G, Krishnadasan A, Gorwitz R, Fosheim G, Mcdougal L, Carey R and Talan D (2006) Methicillin-resistant Staphylococcus aureus infections among in the emergency department. N Engl J Med 355: 666-674.

Pandey S, Raza MS and Bhatta CP (2012) Prevalence and Antibiotic Sensitivity Pattern of MethicillinResistant- Staphylococcus aureus in Kathmandu Medical College, Teaching Hospital. Journal of Institute of Medicine 34: 13-17.

Rao S (2009) Methicillin Resistant Staphylococcus aureus: Available at: www.microrao.com Accessed March, 2009.

Rijal KR, Shrestha N, Pahari N, Shrestha B, Paudel B, Nepal A, Ghimire P and Rijal B (2008) Methicillin resistant Staphylococcus aureus in patients visiting
Western region hospital. Journal of Institute of Medicine 30 (1):21-25.

Shrestha B, Pokhrel BM, Mohapatra TM (2009) Characterization of nosocomial isolates of Staphylococcus aureus with reference to MRSA. J Infect Dev Ctries 3:53-56

Shrestha J, Prajapati K, Panta O, Poudel P and Khanal S (2018) Methicillin Resistant Staphylococcus aureus Isolated from Wound Infections. Tribhuvan University Journal of Microbiology 5: 19-24.

Stapleton PD and Taylor PW (2002). Methicillin resistance in Staphylococcus aureus. US National Library of Medicine 85: 57-72.

Thapa KB (2011) Methicillin Resistant Staphylococcus aureus (MRSA) in clinical samples and nasal screening for MRSA carriage among healthy carriers in hospital setting. M.Sc. Dissertation submitted to Central Department of Microbiology, Tribhuwan University.

Thapa S, Pant DK, Ghimire P, and Thapa PB (2008) Prevalence of Methicillin Resistant Staphylococcus aureus (MRSA) in children. I Nepal Health Res Counc 6: 38-41.

Tiwari HK, Das AK, Sapkota D, Sivrajan KK, Pahwa VK (2009) Methicillin resistant Staphylococcus aureus: prevalence and antibiogram in a tertiary care hospital in western Nepal. J Infect Dev Ctries 3: $681-684$

Voss A and Doebbeling BN (1995) The worldwide prevalence of methicillin resistant Staphylococcus aureus. Int J Antimicrob Agents 5: 101-106. 